\title{
Preface
}

\section{Ambulatory Anesthesia}

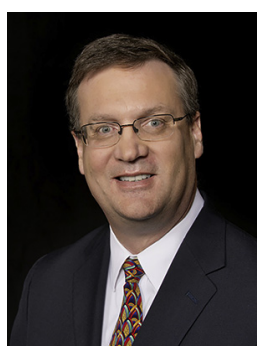

Michael T. Walsh, MD

Editor

\section{We're all ambulatory anesthesiologists now!}

This quote originated with Keynesian economic theory in the 1970s and has a couple of meanings: solidarity, to be sure, but also a sense of inevitable dominance. I think both meanings help illustrate my point when it comes to ambulatory anesthesia. First, the percentage of ambulatory surgical procedures and anesthetics continues to grow with greater than $60 \%$ of all surgeries in the United States classified as ambulatory. Office-based procedures have grown even faster. As of $2014,11 \%$ of the estimated 885 million office visits in the United States were for a surgical procedure. Of course, not everyone is literally practicing ambulatory anesthesia every day, but many subspecialties and procedures once thought to be confined to the inpatient realm are beginning to shift to same-day surgery and 23-hour stay. Outpatient minimally invasive cardiac and vascular procedures, total joint arthroplasty, spine surgery, and minimally invasive neurosurgery are seeing a growing migration toward ambulatory surgery. The nonoperating room procedural practice is seeing explosive growth, and ambulatory patients make up the lion's share.

The dominance of ambulatory surgery and anesthesia is not just about the numbers. More importantly, it's about the mindset; the idea that all anesthetics should return patients to their preoperative functional status as quickly as possible and with minimal pain or side effects. Ambulatory anesthesia was an early proponent of minimizing opioids and utilizing multimodal therapy for pain control and PONV treatment. The enhanced recovery protocols currently popular for inpatient procedures that emphasize early discharge and same-day ambulation and oral intake are a perfect example of the ambulatory philosophy taken to the next level. We can't discharge all our patients in the first day (yet!), but our anesthetic approach continually strives to reach that lofty goal, and as minimally invasive surgical techniques and pain control continue 
to improve, someday, the promise of nearly all anesthesiologists being ambulatory anesthesiologists may come to fruition.

This issue of Anesthesiology Clinics focuses on the present state of ambulatory anesthesia; however, because of ambulatory's broad reach, I think the topics will be of interest to all anesthesiologists. Clinical topics begin with an article on preoperative evaluation, with special emphasis on cardiac conditions and chronic pain patients. A separate article on obesity and obstructive sleep apnea explores the current data and latest guidelines for these commonly encountered conditions. Other important clinical articles include an ambulatory-based focus on enhanced recovery and emergency response. Specific patient groups are discussed in articles on total joint arthroplasty, regional anesthesia, pediatrics, and GI/NORA. Finally, safety in office-based anesthesia and the special concerns of pediatric dental anesthesia rounds out the clinical section.

In 1970, anesthesiologists John Ford and Wallace Reed opened the first free-standing ASC, and ambulatory anesthesiologists have been involved in the practice management side of same-day surgery ever since. While not every ambulatory anesthesiologist holds the actual title of medical director, most of us practice with that mindset: always looking for ways to improve quality, efficiency, and patient satisfaction. It is hoped that the practice management articles focusing on quality, outcomes, and payment will make that job easier. The article on medical director issues offers a nice synopsis and invaluable thoughts on leadership.

I want to thank all of the authors for their time and talents in contributing to this issue. Their dedication and expertise really shine through and it is hoped will offer valuable tips and strategies to help you improve the care of your patients, no matter where you practice.

Michael T. Walsh, MD Department of Anesthesiology \&

Perioperative Medicine Mayo Clinic

100 1st Street SW Rochester, MN 55901, USA 